\title{
Revised and Improved Implementation of the Spur Involute Gear Dynamical Model
}

\author{
Ivan Kosenko* Ilya Gusev** \\ *Dorodnitsyn Computing Center of Russian Academy of Sciences, Department of Mechanics \\ Moscow, 119333, Russia \\ ${ }^{* *}$ Russian State University of Tourism and Service, Department of Natural and \\ Engineering Sciences, Cherkizovo-1, Moscow region, 141221, Russia
}

\begin{abstract}
An improved model having new, more realistic, properties is constructed with use of previously implemented approach for building up a model of the spur involute gear dynamics. First of all, an algorithm for contact tracking of cylindrical surfaces directed by involutes was rearranged. This algorithm is "simply" reduced to tracking the two involutes. A result is that common line normal to these contact curves always coincides with the line of action. This property permits obtaining direct simple formulae for contact computations.
\end{abstract}

A backlash in gearbox is also taken into account in the model under consideration. This means that a loss of contact between the teeth is possible as gearwheels rotate. This may then cause an appearance of a contact patch during the reversal. Furthermore, a dynamical reasons may force the mesh process to return to the former mode of the forward stroke and so fourth. All such scenarios for switching modes are implemented in the model in a unified way.

A time overlapping of contacts between teeth pairs is used to ensure the mesh reliability. This property is also implemented in the described dynamical model. New contact of the next pair of teeth arises and starts its motion along the line of action before the old contact leaves this line at the point of teeth disengagement.

Keywords: spur gear; involute; mesh properties; tracking algorithm; mesh ratio; multiple contact; backlash

\section{Introduction}

One can highlight two poles among all approaches to computer modeling and simulation of the gear dynamics. Computational algorithms of high accuracy are relocated at one end of the corresponding scale. These algorithms take into account elasto-plastic properties of the material that the contacting bodies are made of, plus a variety of boundary conditions of different types [1]. Such high accuracy simulation models simultaneously require significant computational resources. One might point out different simplified models, see e. g. [2], on the other end of the scale. These models provide the highest efficiency.

The compromise model presented in [3] might be improved upon in a way so as to take into account essential properties of real gear: (a) backlash, (b) contact multiplicity. The latter property is always provided in real gears in order to prevent jamming in teeth. In addition, the contact tracking algorithm turned out to be simplifiable and simultaneously essentially accelerateable in the case of the involute mesh. For definiteness, we use the Johnson [4] model for the cylindrical bodies contact as was previously done [3] for the case of spur mesh.

\section{Preliminaries}

Using methodics $[5,6]$ previously developed for computer modeling of the rigid bodies 3D-motions let us consider planar motion for bodies of cylindrical shape, denoted as $A$ and $B$ in our case, in the plane orthogonal to generatrix of cylinders. We connect this plane with an additional body $C$, see Figure 1, an auxiliary frame $O_{C} x y z$ of coordinates is assumed to be rigidly connected with that latter body in a way such that cylindrical generatrix is always orthogonal to the axis $O_{C} z$. One might express this latter requirement using the following geometrical conditions: $\mathbf{k}_{\alpha}=\mathbf{k}_{C}(\alpha=A, B)$, where $\mathbf{k}_{\alpha}(\alpha=A, B, C)$ are the unit vectors defining the axes $O_{\alpha} z$ connected with the bodies $\alpha=A, B$. To ensure the motion of the bodies in the plane parallel 
to the plane $O_{C} x y$ let us require a fulfillment of yet two more algebraic conditions for the bodies $A$ and $B$ $z$-coordinates: $z_{O_{A}}=$ const, $z_{O_{B}}=$ const. All the coordinates are given with respect to (w. r. t.) the system $O_{C} x y z$.

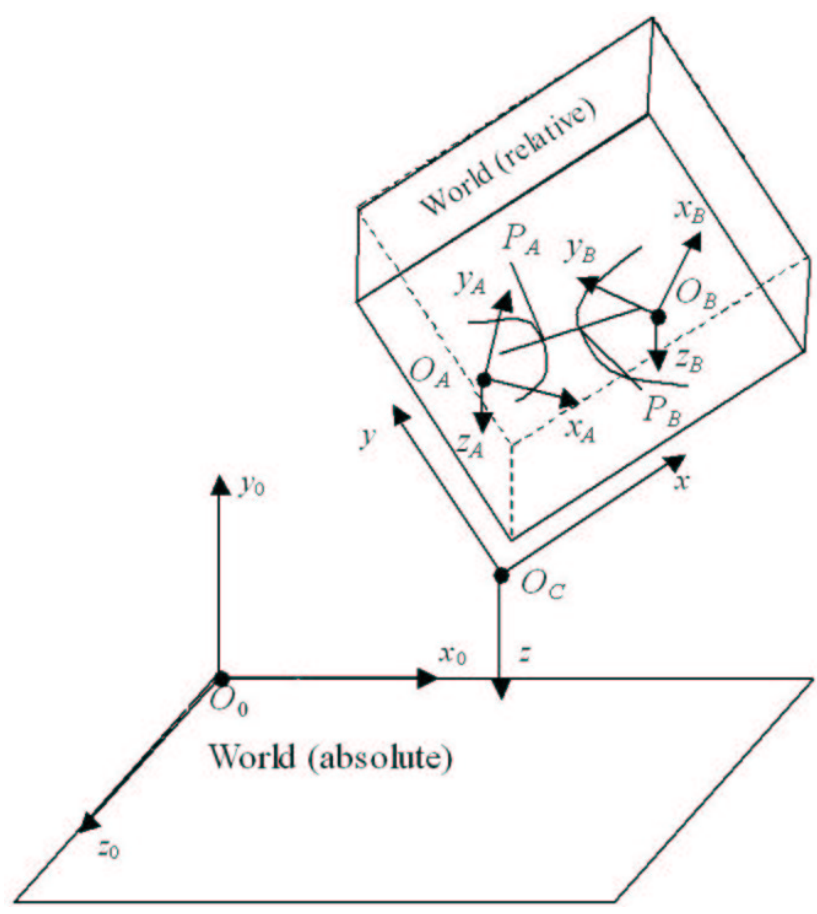

Figure 1: Coordinate systems for the model: (a) base frame of reference $O_{0} x_{0} y_{0} z_{0}$; (b) the gearbox housing coordinate system $O_{C} x y z$; (c) the pinion coordinate system $O_{A} x_{A} y_{A} z_{A}$; (d) the gear coordinate system $O_{B} x_{B} y_{B} z_{B}$

One can easily implement algebraic equations enumerated above in implicit form. To fix the bodies $A$ and $B$ w. r. t. the body $C$ one can use, for instance, constraints of the joint type $[5,6]$. In this case the body $C$ itself performs arbitrary 3D-motions being regarded as a convective motion w. r. t. certain inertial frame of reference. Thus calling the body $C$ as the reductiongear housing is quite natural, if the bodies $A$ and $B$ are models of gearwheels. After the reduction to the plane $O_{C} x y$ performed above building up a technique for the cylindrical bodies using 2D-geometry properties [3] is quite natural as well.

Note that all the bodies $A, B, C$ in the model perform their 3D-motions according to the spatial dynamics of rigid body encapsulated in the corresponding base class. And relative cylindrical symmetry of bodies $A$ and $B$ w. r. t. the body $C$ is kept due to the reaction forces between them. These forces are generated dynamically in an acausal mode due to kinematical constraints encapsulated in a contact class, rather a tem- plate, being further constructed in this paper.

\section{Account of the backlash}

First of all, let us simplify and as a consequence essentially accelerate a performance of the previously implemented algorithm of the contact tracking for two involute surfaces of the teeth pair at the contact for the spur gear meshing. Such a simplification allows us building up the mesh model quite easily for the mesh ratio greater than one, and simultaneously accounting for the backlash.

As was found earlier [3] that in the case of the involute mesh the sought points $P_{A}$ and $P_{B}$, see Figure 1, lying both on the perpendicular common for involutes of gearwheels teeth in vicinity of contact, are located simultaneously on the mesh line of action $K_{A} K_{B}$, see Figure 2. Evidently, the common perpendicular mentioned above also coincides with the line of action $K_{A} K_{B}$. Thus, from the geometric point of view the point $P_{A}$ lies permanently in time on the intersection of the gearwheel tooth involute and the line $K_{A} K_{B}$. Similar statement takes place for the point $P_{B}$ : it lies on the intersection of the gearwheel $B$ tooth involute and the same line of action $K_{A} K_{B}$.

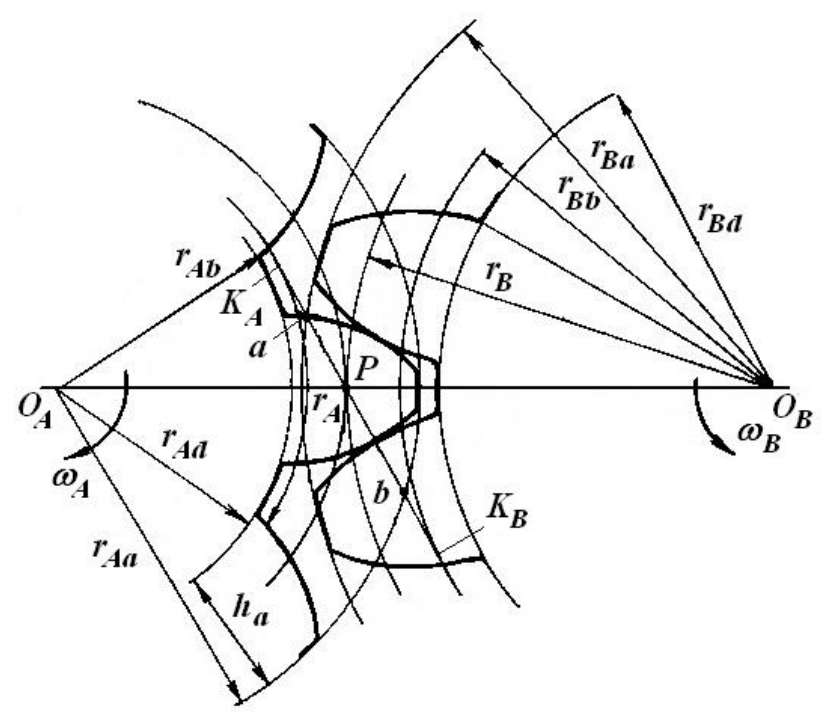

Figure 2: Gear mesh for forward stroke

Thus in case of involutes computing coordinates $s_{A}$ and $s_{B}$ of points $P_{A}$ and $P_{B}$ respectively on the strait line $K_{A} K_{B}$ replacing a cumbersome algorithm using differential-algebraic equations is sufficient for contact tracking. One can compute coordinates $s_{A}, s_{B}$ with an extremely simple procedure, see Figure 3.

Let the coordinates $s_{A}, s_{B}$ denote the distances from 


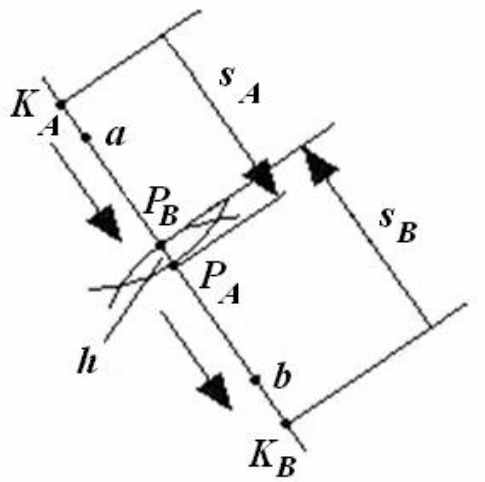

Figure 3: Contact tracking coordinates

the respective points $K_{A}, K_{B}$. We assume values of $s_{A}$, $s_{B}$ at these source points being set to zero. Thus for $s_{A}+s_{B} \geq L=\left|K_{A} K_{B}\right|$ a contact takes place, and for $s_{A}+s_{B}<L$ the contact is absent. In the first case the depth $h$ of the bodies mutual penetration is computed by the simple formula $h=L-s_{A}-s_{B} \leq 0$. Left arrows in Figure 3 show a direction in which the contact patch moves for the forward mode and as pinion rotates clockwise.

When computing the value $h$ the pair of teeth being in contact is under analysis. In case of forward stroke we assume for definiteness that the wheel $A$, pinion, rotates clockwise while the wheel $B$, gear, supposed to rotate counterclockwise. The angles $\varphi_{A}, \varphi_{B}$ of rotation of the bodies $A, B$ respectively are defined by the axis $O_{C} x$ of the gearbox housing and by the axes beginning from bodies' points $O_{A}, O_{B}$ and going through the points of their base circles where corresponding involutes "grow", see Figure 2.

Furthermore, if the wheel $A$, for definiteness, rotates such that the angle $\varphi_{A}$ appears outside its admissible limits (being defined below) then the model generates an event corresponding to fulfilment of the condition $\varphi_{A} \notin\left[\varphi_{A \text { min }}, \varphi_{A \text { max }}\right]$. In such a case the values of angles $\varphi_{A}=\varphi_{A}^{-}, \varphi_{B}=\varphi_{B}^{-}$are to be automatically corrected according to equations (we assume that contact of the forward stroke exists currently):

$$
\varphi_{A}^{+}=\varphi_{A}^{-}+m \Delta \varphi_{A}, \quad \varphi_{B}^{+}=\varphi_{B}^{-}-m \Delta \varphi_{B}
$$

for the case of $\varphi_{A}<\varphi_{A \min }$ and equations:

$$
\varphi_{A}^{+}=\varphi_{A}^{-}-m \Delta \varphi_{A}, \quad \varphi_{B}^{+}=\varphi_{B}^{-}+m \Delta \varphi_{B}
$$

for the case of $\varphi_{A}>\varphi_{A \max }$. Here $\Delta \varphi_{A}, \Delta \varphi_{B}$ are angular widths per one tooth of the wheels $A$ and $B$ respectively; $m$ is the mesh multiplicity (the least integer greater than the mesh ratio). Note that the angles $\varphi_{A}$ and $\varphi_{B}$ are not exactly the bodies angles of rotation.
They are indeed the angles of rotation for wheels' teeth w. r. t. the axis $O_{C} x$. These teeth are supposed to lie currently in the zone of possible contact. This zone is defined by the condition $\varphi_{A} \in\left[\varphi_{A \min }, \varphi_{A \max }\right]$.

Formulae from above have to simply switch contact in the same sense as it was arranged in [3]. The following approximate rule is used: at the very same moment when the contact patch "instantly vanishes" behind an upper or lower limits of admissible segment $\left[\varphi_{A \min }, \varphi_{A \max }\right]$ this patch should appear immediately on the other end of the same segment. For simplicity the wheel $A$ is considered as a "leading" object responsible for the event generation process.

Thus a current contact object of the model "jumps" to the next pair of teeth over $m-1$ pairs being currently in contact if the object individual angle $\varphi_{A}$ of tooth rotation goes out of its admissible limits. Recall that $m$ is the mesh multiplicity, and in general we assume $m \geq 1$.

Limit values $\varphi_{A \min }, \varphi_{A \max }$ for angle of inclination of an involute at contact for the current pair of teeth are computed with natural restrictions being imposed on the contact area. Minimal value $\varphi_{A \text { min }}$ corresponds to the final point $b$ of contacting along the line of action for the case of forward stroke, see Figure 2. One can see easily that the value $\varphi_{A \text { min }}$ is computed by the formula

$$
\varphi_{A \min }=\alpha_{w}-\frac{\left|K_{A} a\right|+|a b|}{r_{A b}}
$$

corresponding to the selection of points $a$ and $b$ where the contact process of starts and ends respectively. One might find details for such matching in [3]. Here $\alpha_{w}$ is the pressure angle, and $r_{A b}$ is the wheel $A$ base circle radius. Equation (1) has a simple geometrical explanation, see Figure 4. Indeed, consider the pinion base circle. Its arc length from the point $K_{A}$ downwards to the position corresponding to the angle $\varphi_{A \max }$ should be equal to the segment $\left[K_{A}, b\right]$ length according to the known involute property. This circumstance leads immediately to property (1). We recall that the point $b$ on the gear mesh line of action defines the position where contact patch vanishes.

If the wheel $A$ angular position $\varphi_{A}=\varphi_{A \text { min }}$ corresponds to the instant for contact finishing then the angle $\varphi_{A}=\varphi_{A \max }$ has to correspond to this process beginning for the current pair of teeth. One easily sees that the assumption $\varphi_{A \max }=\varphi_{A \text { min }}+m \Delta \varphi_{A}$ has to hold.

Similarly, obtaining formulae for computation of 


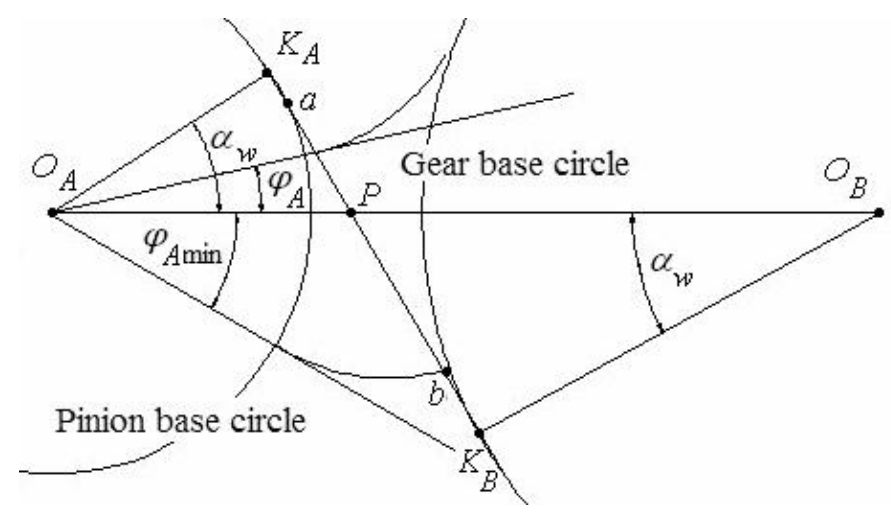

Figure 4: Limit angle $\varphi_{A \min }$

the points $P_{A}, P_{B}$ coordinates $s_{A}, s_{B}$ as

$$
\begin{aligned}
& s_{A}=\left\{\begin{array}{cl}
r_{A b}\left(\alpha_{w}-\varphi_{A}\right) & \text { for } \varphi_{A}<\alpha_{w}, \\
0 & \text { for } \varphi_{A} \geq \alpha_{w},
\end{array}\right. \\
& s_{B}=\left\{\begin{array}{cl}
r_{B b}\left(\alpha_{w}+\pi-\varphi_{B}\right) & \text { for } \varphi_{B}<\pi+\alpha_{w}, \\
0 & \text { for } \varphi_{B} \geq \pi+\alpha_{w},
\end{array}\right.
\end{aligned}
$$

where $r_{B b}$ is the wheel $B$ base circle radius, is not very difficult.

One has to provide additional contact (between wheels $A$ and $B$ ) objects to take into account all the possible contacts of teeth pairs if the mesh ratio is greater than one. To simulate the gearbox forward stroke one has to provide generally $m$ instances of such contact objects.

Furthermore, clearly, if contact of teeth in the forward stroke vanishes then it is almost evident that contact of reversal arises. This latter arises between the teeth pair closest to contact lost before and being located through the tooth trough on the involutes of the teeth sides previously unused in the forward stroke mode.

To simulate the reversal one has to use line of action derived from the line of Figure 2 by mirroring it w. r. t. the axis connecting points $O_{A}$ and $O_{B}$. All the mesh geometric properties considered for the forward stroke are mirrored for the case of reversal. In particular, coordinates $s_{A}$ and $s_{B}$ for this case have expressions

$$
\begin{aligned}
& s_{A}=\left\{\begin{array}{cl}
r_{A b}\left(\alpha_{w}+\varphi_{A}\right) & \text { for } \varphi_{A}>-\alpha_{w}, \\
0 & \text { for } \quad \varphi_{A} \leq-\alpha_{w},
\end{array}\right. \\
& s_{B}=\left\{\begin{array}{cl}
r_{B b}\left(\alpha_{w}-\pi+\varphi_{B}\right) & \text { for } \varphi_{B}>\pi-\alpha_{w}, \\
0 & \text { for } \varphi_{B} \leq \pi-\alpha_{w} .
\end{array}\right.
\end{aligned}
$$

Note that what we have meant under "the forward stroke" or "reversal" is not a kinematical property whether to rotate clockwise or counterclockwise but it is a dynamical property switching into work/contact between driving/driven surfaces of teeth. Thus, we will see the forward stroke in cases of the pinion $A$ clockwise accelerated and counterclockwise decelerated rotation. Similarly, reversal takes place in general if pinion $A$ accelerates when rotating counterclockwise and decelerates simultaneously rotating clockwise. Simplifying formulations let us call the rotation with line of action shown in Figure 2 as the forward stroke. Likewise, the rotation with line of action mirrored w. r. t. the axis $O_{A} O_{B}$ of Figure 2 be called as reversal. The reversal requires correct switching between pairs of teeth, as well as, it was implemented for the forward stroke.

When contacting in reversal mode switching of the teeth pairs takes place if the contact patch leaves the segment $a^{\prime} b^{\prime}$ of line of action $K_{A}^{\prime} K_{B}^{\prime}$, see Figure 5 , or by the point $a^{\prime}$ or through the point $b^{\prime}$. For that one has to apply the same relations as above replacing the segment $\left[\varphi_{A \text { min }}, \varphi_{A \text { max }}\right]$ of admissible values for the angle $\varphi_{A}$ by the segment $\left[\varphi_{A \text { min }}^{\prime}, \varphi_{A \text { max }}^{\prime}\right]$ for the angle $\varphi_{A}^{\prime}$ such that $\varphi_{A \text { min }}^{\prime}=-\varphi_{A \max }, \varphi_{A \max }^{\prime}=-\varphi_{A \min }$.

\section{Case of multiple contact}

Previously mesh ratio was supposed equal to one in the simplified model of the gear mesh [3]. This means that exactly at the moment of contact loss at the point $b$ new contact at the point $a$ arises. Such an arrangement leads frequently to a low reliability of a gearbox as well-known however in practice, mostly due to jamming caused by manufacturing errors. Due to this reason ensuring a reliable gearbox work one provides overlapping for time intervals of contacts in teeth pairs. Namely, new contact at the point $a$ arises earlier than the current contact vanishes at the point $b$.

Let us return to the example being analysed in [3] where a virtual setup for computational experimenting was constructed, see Figure 6 and also Figure 1 for ge- 


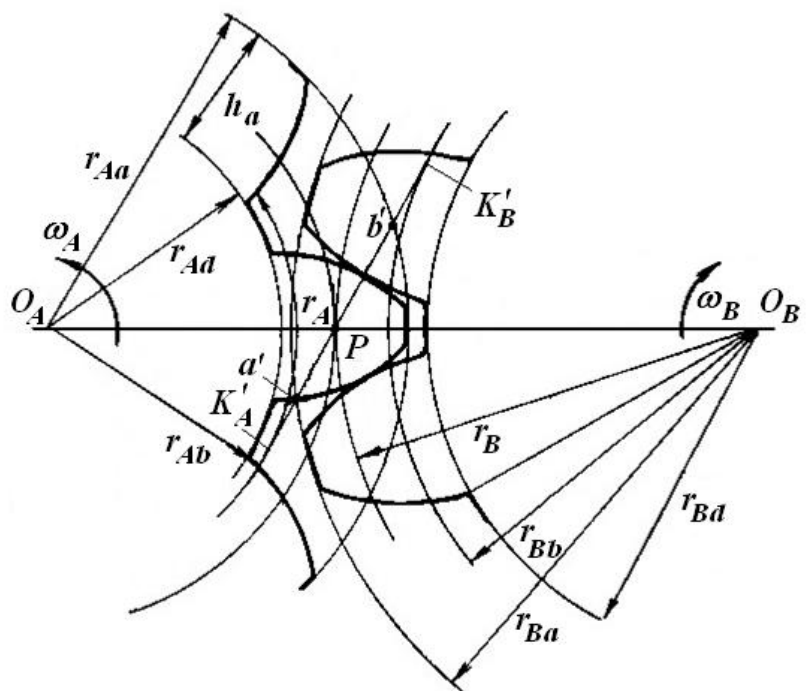

Figure 5: Gearmesh for reversal mode

ometry of the whole construct. This setup consists of two gearwheels: pinion $A$ and gear $B$. For simplicity we assume the gearbox housing $C$ fixed w. r. t. the base body of a whole multibody system. Furthermore, origin $O_{C}$ of an inertial frame $O_{C} x y z$ of reference coincides with the pinion $A$ center where the revolute joint which connects bodies $A$ and $C$ is located. The gear $B$ center locates on the horizontal axis $O_{C} x$. There exists the second revolute joint connecting the body $B$ and auxiliary slider $S$. The slider $S$ in turn may freely slip w. r. t. the body $C$ along the axis $O_{C} x$. This slipping however is decelerated by a spring of very large stiffness. The spring connects the bodies $C$ and $S$ thus providing a compliance between the bodies $B$ and $C$ through the intermediate slider $S$. This compliance has direction along the line $O_{A} O_{B}$ connecting the wheels centers and coinciding with the axis $O_{C} x$. Such a construct prevents static indefiniteness in the model for the case of the rigid point-contact in the gearmesh of the wheels $A$ and $B$.

We define in the model the following independent parameters:

- $z_{A}=20$ is number of the pinion teeth;

- $z_{B}=30$ is number of the gear teeth;

- $r_{A}=0.2 \mathrm{~m}$ is the pinion pitch circle radius.

Other (dependent) geometric parameters are computed as follows

- $n=z_{B} / z_{A}$ is the transmission ratio;

- $r_{B}=n r_{A}$ is the gear pitch circle radius;
- $\Delta \gamma_{A}=2 \pi / z_{A}, \Delta \gamma_{B}=2 \pi / z_{B}$ are the pitch angles of the pinion and gear.

For further definition of the gear mesh choosing the pressure angle value is important. This value has to satisfy the condition $\alpha_{w}>\alpha_{w \text { inf }}$, where $\alpha_{w \text { inf }}=\inf \alpha_{w}$ is the lower bound for all possible pressure angles which are admissible by parameters selected above. One can compute this bound according to the formula

$$
\alpha_{w \text { inf }}=\arctan \frac{2 \pi}{z_{A}(1+n)} .
$$

The lower bound obtained above is a simple consequence of the mesh natural condition

$$
\left|\overrightarrow{K_{A} K_{B}}\right|>|\overrightarrow{a b}|
$$

For definiteness let us choose the value

$$
\alpha_{w}=2.8 \alpha_{w \text { inf }} .
$$

Furthermore, with the help of the pressure angle value and the value of the transmission ratio we can compute all the geometric parameters needed shown in Figure 2. Firstly of all one can obtain radii of base circles as

$$
r_{\alpha b}=r_{\alpha} \cos \alpha_{w} \quad(\alpha=A, B) .
$$

Then one can compute full length of the line of action in the following way

$$
\left|\overrightarrow{K_{A} K_{B}}\right|=r_{A}(1+n) \sin \alpha_{w} .
$$

At the same time, the length of any segment of contact $[a, b] \subset\left(K_{A} K_{B}\right)$ along this line is exactly the length of the base circle arc corresponding to the pitch angle $\Delta \gamma_{A}$ or $\Delta \gamma_{B}$ for any wheel of the gearbox. Thus we have

$$
|\overrightarrow{a b}|=r_{\alpha b} \Delta \gamma_{\alpha} \quad(\alpha=A, B) .
$$

One easily computes the distance between wheels centers as $L=r_{A}+r_{B}$. For computing initial conditions in the model performing additional calculations is necessary. Suppose for definiteness that the coordinate system $O_{C} x_{C} y_{C} z_{C}$ has its origin at the point $O_{A}$ of the pinion $A$ center: $O_{C}=O_{A}$, so that these points initial absolute coordinates coincide. Thus

$$
\mathbf{r}_{O_{C}}=\mathbf{r}_{O_{A}}=(0,0,0)^{T},
$$

and the initial position of the gear center is defined by

$$
\mathbf{r}_{O_{B}}=(L, 0,0)^{T} .
$$




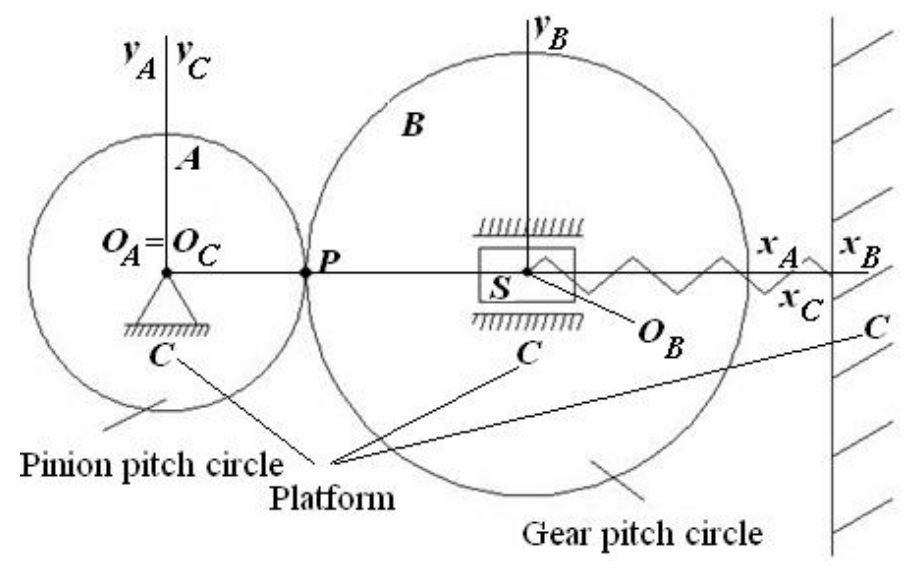

Figure 6: Virtual setup for computational experiments

Initial positions of the points $K_{A}$ and $K_{B}$ are computed by vector formulae

$$
\begin{aligned}
& \mathbf{r}_{K_{A}}=\mathbf{r}_{O_{A}}+r_{A b}\left(\cos \alpha_{w}, \sin \alpha_{w}, 0\right)^{T}, \\
& \mathbf{r}_{K_{B}}=\mathbf{r}_{O_{B}}-r_{B b}\left(\cos \alpha_{w}, \sin \alpha_{w}, 0\right)^{T}
\end{aligned}
$$

being deduced easily. Furthermore, a directing vector for the line of action is defined as $\overrightarrow{K_{A} K_{B}}=\mathbf{r}_{K_{B}}-\mathbf{r}_{K_{A}}$. So that the contact starting point $a$ initial position may be defined as

$$
\mathbf{r}_{a}=\mathbf{r}_{K_{A}}+\frac{1}{2}\left(\left|\overrightarrow{K_{A} K_{B}}\right|-|\overrightarrow{a b}|\right) \frac{\overrightarrow{K_{A} K_{B}}}{\left|\overrightarrow{K_{A} K_{B}}\right|}
$$

and the initial position for the point $b$ of contact finishing as

$$
\mathbf{r}_{b}=\mathbf{r}_{a}+\frac{|\overrightarrow{a b}|}{\left|\overrightarrow{K_{A} K_{B}}\right|} \overrightarrow{K_{A} K_{B}}
$$

Let us take into account that the distance between the points $a$ and $O_{B}$ is exactly equal to the addendum circle radius $r_{B a}$ for the wheel $B$, and the initial distance from the point $b$ to $O_{A}$ is equal to the addendum circle radius $r_{A a}$ for the wheel $A$. Namely

$$
r_{A a}=\left|\mathbf{r}_{b}-\mathbf{r}_{O_{A}}\right|, \quad r_{B a}=\left|\mathbf{r}_{a}-\mathbf{r}_{O_{B}}\right| .
$$

To ensure overlapping of the mesh cycles for wheels with transmission ratio $n=3 / 2$ let us consider the case with $z_{A}=22$ and $z_{B}=33$ providing the same transmission ratio. Note that the real angular widths $\Delta \varphi_{A}=\pi / 11$ and $\Delta \varphi_{B}=2 \pi / 33$ for teeth become less than their nominal, pitch, widths $\Delta \gamma_{A}=\pi / 10$ and $\Delta \gamma_{B}=2 \pi / 30$.

Simultaneous coexistence of two contacts in the model obtained requires, both in the forward stroke and in reversal, the use of four contact objects in the mesh computer model - two for the forward stroke plus two for reversal. Visual model of the experimental setup is represented in Figure 7. Here Contact $f 1$ and Contactf 2 are objects for the forward stroke, and Contactb1 and Contactb2 are ones for reversal. Thus as a result models for the pinion, the left wheel object LeftWheel, and gear, the right wheel object RightWheel, each has four input ports for information about wrenches arising at patches of an elastic contacts. Object Platform simulates dynamics of the base body (absolute world), gearbox housing $C$ (relative world), having a predefined motion, resting in our case. Thus two mentioned worlds coincide for the model of Figure 7.

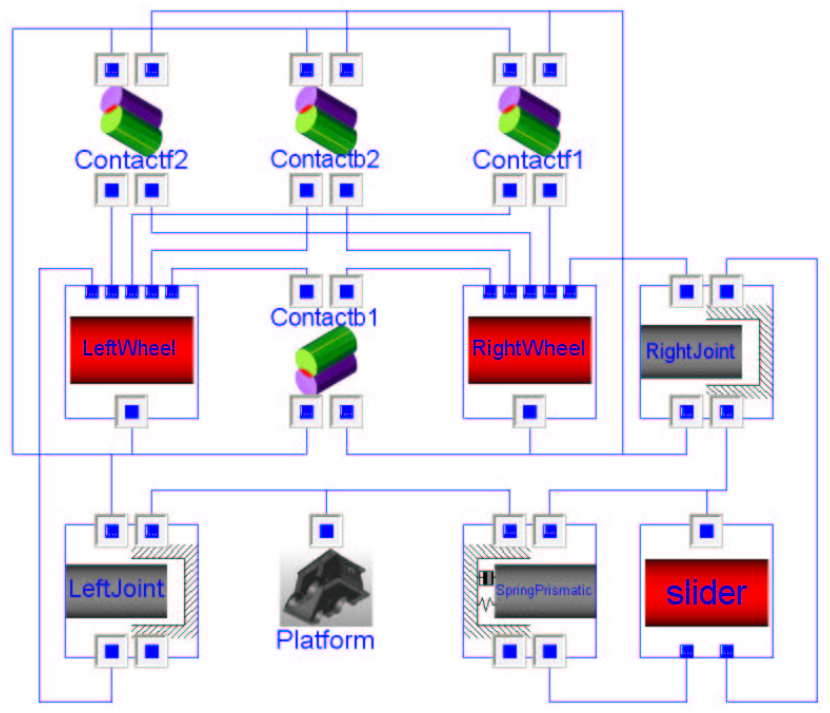

Figure 7: The testbench visual model

Note that each contact object mentioned above works virtually independently. Coordination of their on/off switching is achieved using proper and correct selection of initial conditions for the state variables inside contact objects. In case of our dynamical example 
these conditions are defined in the following way.

Angular velocities of wheels are assumed to be zero. For definiteness we also suppose that the body $A$ axis $O_{A} x_{A}$ goes through the involute root point lying on the pinion base circle. This involute defines exactly the tooth surface, and initially it goes through the point $a$ thus starting a contact. Likewise, the axis $O_{B} x_{B}$ of the wheel $B$ initially goes through the gear $B$ involute root point.

At initial instant of time one pair of the wheels $A$ and $B$ teeth is supposed to have contact at the point $a$, see Figure2, with a zero depth of mutual penetration. For definiteness this contact is supposed to be in the forward stroke mode. One can easily conclude from Figure 2 that in initial position of teeth for angles of inclination $\varphi_{A}, \varphi_{B}$ of radius vectors for the involute root points, lying on the base circles, the equations

$$
\begin{aligned}
& \varphi_{A_{0}}^{f_{1}}=\operatorname{atan} 2\left(a_{y}, a_{x}\right)-\theta_{A}, \\
& \varphi_{B}^{f_{1}}=\operatorname{atan} 2\left(a_{y}-y_{C_{B}}, a_{x}-x_{C_{B}}\right)-\theta_{B},
\end{aligned}
$$

where $\theta_{A}, \theta_{B}$ are the point $a$ polar angles on teeth involutes, hold. These angles are computed in the form

$$
\begin{aligned}
\theta_{A} & =\frac{\sqrt{|\mathbf{a}|^{2}-r_{A b}^{2}}}{r_{A b}}-\arccos \left(\frac{r_{A b}}{|\mathbf{a}|}\right), \\
\theta_{B} & =\frac{\sqrt{\left|\mathbf{a}-\mathbf{r}_{C_{B}}\right|^{2}-r_{B b}^{2}}}{r_{B b}}-\arccos \left(\frac{r_{B b}}{\left|\mathbf{a}-\mathbf{r}_{C_{B}}\right|}\right) .
\end{aligned}
$$

Thus wheels initial angles of inclination in the object Contactf1 are defined by formulae (2). Furthermore, for the mesh ratio being greater than one if one pair of teeth starts contact at the point $a$ then another neighbour pair being ahead of the previous one will have a contact somewhere on the segment $[a, b]$. This latter contact is supposed to be defined in the object Contact $f 2$. Initial values of auxiliary angles $\varphi_{A}, \varphi_{B}$ defining the angles of involute rotation for the forward stroke (or reversal) and being respectively in segments $\left[\varphi_{A \min }, \varphi_{A \max }\right],\left[\varphi_{B \min }, \varphi_{B \max }\right]$ are to be distanced from the angles of the object Contact 1 exactly by the tooth angular width (which is smaller than the angular pitch of the gear mesh under simulation). Namely, the following formulae

$$
\varphi_{A_{0}}^{f_{2}}=\varphi_{A}^{f_{1}}-\Delta \varphi_{A}, \quad \varphi_{B}^{f_{2}}=\varphi_{B}^{f_{1}}-\Delta \varphi_{B}
$$

are to be satisfied.

An initial data selection for the objects Contactb1, Contactb2 servicing the reversal is not so evident. Indeed, involutive surfaces of the teeth pair being tracked by the object Contactb1 will be situated on the same teeth as the surfaces being tracked by the object Contactf2. The only difference is that they should be relocated on other sides of the teeth mentioned, see Figure 2. So from geometrical point of view contact of reversal being tracked by the object Contactb1 on initial stage of motion should be located between the contacts of the forward stroke being tracked by the objects Contact 1 and Contact $f 2$.

Note that for a particular tooth the radius vectors of the involute root points (lying on the base circles) of its two sides rotate w. r. t. each other exactly by an angular width of one tooth without accounting for the tooth trough. Note that all angular widths are to be counted along the arcs of the base circle. Denote these angular widths of teeth by $t_{A \text { wid }}, t_{B \text { wid. }}$. Then one can compute initial data in the object Contactb1 by the formulae

$$
\varphi_{A_{0}}^{b_{1}}=\varphi_{A_{0}}^{f_{2}}+t_{A \text { wid }}, \quad \varphi_{B 0}^{b_{1}}=\varphi_{B 0}^{f_{1}}+t_{B \text { wid }} .
$$

Let us remark here that really at the initial instant of the computational experiment the objects Contactb1, Contactb2 generate zero-valued wrenches of contact forces. All this is due to the contacts absence for reversal mode though objects Contactb1, Contactb2 always continue to track the points $P_{A}, P_{B}$ inside each of them.

The object Contactb2 of the second contact for the reverse mode has the following initial data for the involute angles of inclination (rather angles of inclination of their root points radius vectors for the case $m=2$ )

$$
\varphi_{A_{0}}^{b_{2}}= \begin{cases}c & \text { for } \quad c>\varphi_{A \min } \\ d & \text { for } \quad c \leq \varphi_{A \min }\end{cases}
$$

where $c=\varphi_{A_{0}}^{f_{1}}-2 \Delta \varphi_{A}+t_{A \text { wid }}, d=\varphi_{A_{0}}^{f_{1}}+t_{A \text { wid }}$ and

$$
\varphi_{B_{0}}^{b_{2}}= \begin{cases}q & \text { for } \quad c>\varphi_{A \min } \\ r & \text { for } \quad c \leq \varphi_{A \min }\end{cases}
$$

where $q=\varphi_{B 0}^{f_{1}}+\Delta \varphi_{B}+t_{B \text { wid }}, r=\varphi_{B 0}^{f_{1}}-\Delta \varphi_{B}+t_{B \text { wid }}$.

In the latter equations we take into account the fact that for the case of the mesh multiplicity for the reversal mode there exist several possibilities, two in our example, of contact implementations along the line of action $K_{A}^{\prime} K_{B}^{\prime}$, see Figure 5 .

Ensuring the initial data selection from above in the objects of contact we thus automatically provide correct switching of modes of contact inside the objects and correct tracking for involutes contact switching in the process of wheels rotation. The built up mesh 
model provides a possibility to simulate motions of any type in the gearbox with any combination for contact between teeth. This model enables us able to construct effectively the gearboxes virtual prototypes of any complexity for the case of the spur involute gear.

\section{Behavioral Model of Contact Object}

Let us return to the gearbox visual model presented in Figure 7. It has been built with the help of earlier proposed $[5,6]$ technologies for constructing the physically-oriented models. For each physically implemented contact of the model there exists one object of visual model, see Figure 7. Meanwhile, from the functional viewpoint there is no difference how contacts of specific type, Nos. 1 and 2 for the forward stroke and Nos. 1 and 2 for reversal, are redistributed over an array of unified contact objects. Thus, the same class code is able "to play a role" of contact of any type within the spur involute gear model. In virtue of the circumstances outlined above organizing an array for all four contact objects in virtual model is reasonable. There should also be an array of four connectors reserved for transmitting data of wrenches from contact objects to objects of bodies, the wheels LeftWheel, RightWheel in Figure 7. In this case corresponding wrench ports are to be really arrays of ports [7] in objects LeftWheel and RightWheel.

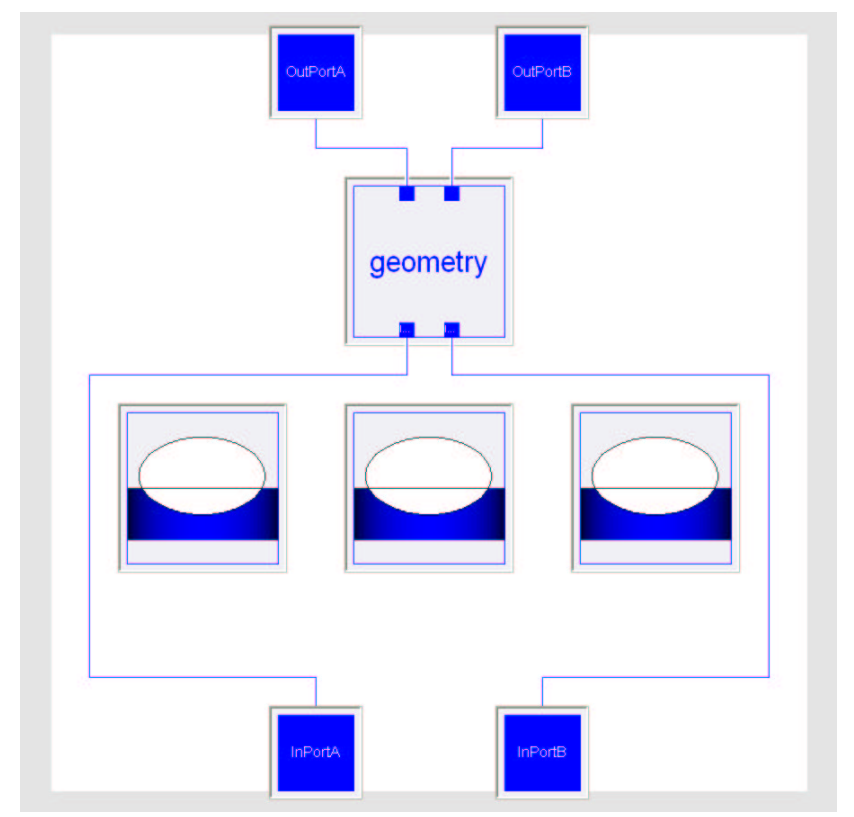

Figure 8: Base template for contact model

Note that according to the approach previously im- plemented in [8] contact objects have a class being a template which has four class parameters responsible for implementation of: (a) geometry of surfaces at contact; (b) model of normal elastic contact forces; (c) model of normal viscous forces; (d) model of tangent (ususally friction) forces. Visual representation of base template with empty sockets for the above four parameters see in Figure 8. The final derived class is shown in Figure 9 with mentioned sockets filled, actually redeclared, respectively by the following model parameters: (a) involute cylinder - involute cylinder; (b) the Johnson contact model for cylindrical bodies; (c) non-linear normal viscous model; (d) simplified Coulomb model of friction for tangent forces.

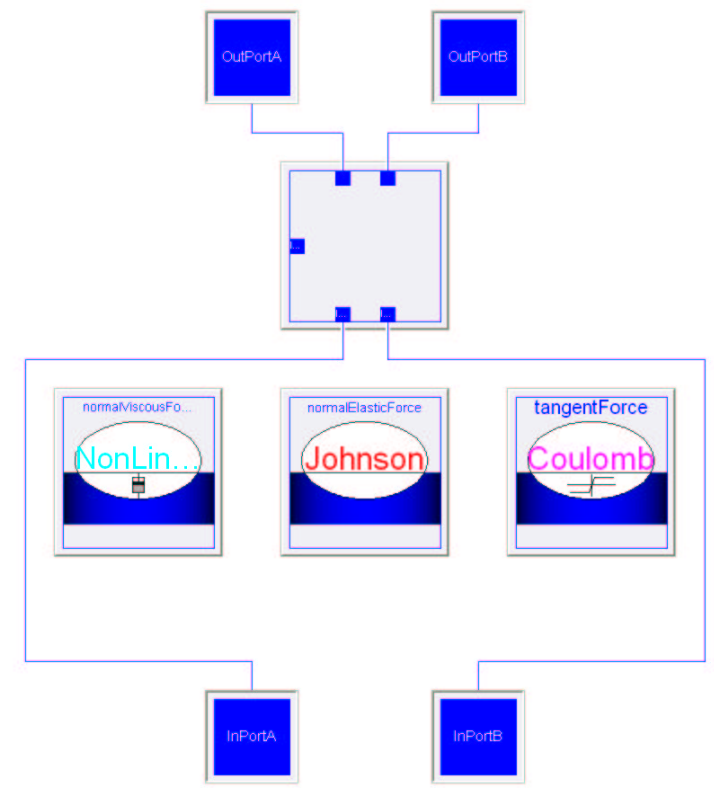

Figure 9: Final derived class for contact model of the example

The angles $\varphi_{A}, \varphi_{B}$ of the wheels relative rotation are fundamental properties of the contact model under consideration. The angle $\varphi_{A}$ is defined clearly in Figure 4. The angle $\varphi_{B}$ has a similar sense for the wheel $B$. These angles remain always bounded throughout simulation: $\varphi_{\alpha} \in\left[\varphi_{\alpha \min }, \varphi_{\alpha \max }\right](\alpha=A, B)$. Simultaneously for derivatives the equations $\dot{\varphi}_{\alpha}(t) \equiv \dot{\psi}_{\alpha}(t)$ are fulfilled almost everywhere for $t \in\left[t_{0}, t_{1}\right]$ where $t_{0}$ is the starting instant of the simulation process, $t_{1}$ is the instant of the simulation finish. Here the values $\psi_{\alpha}(\alpha=A, B)$ are assumed angles of the gearwheels rotation w. r. t. the gearbox housing. The variables $\psi_{\alpha}(\alpha=A, B)$ are defined by dynamical equations of the model. In general the angle $\psi_{\alpha}(t)$ may grow or decrease infinitely. At the same time the angles $\varphi_{\alpha}(t)$ always remain bounded. The property described above 


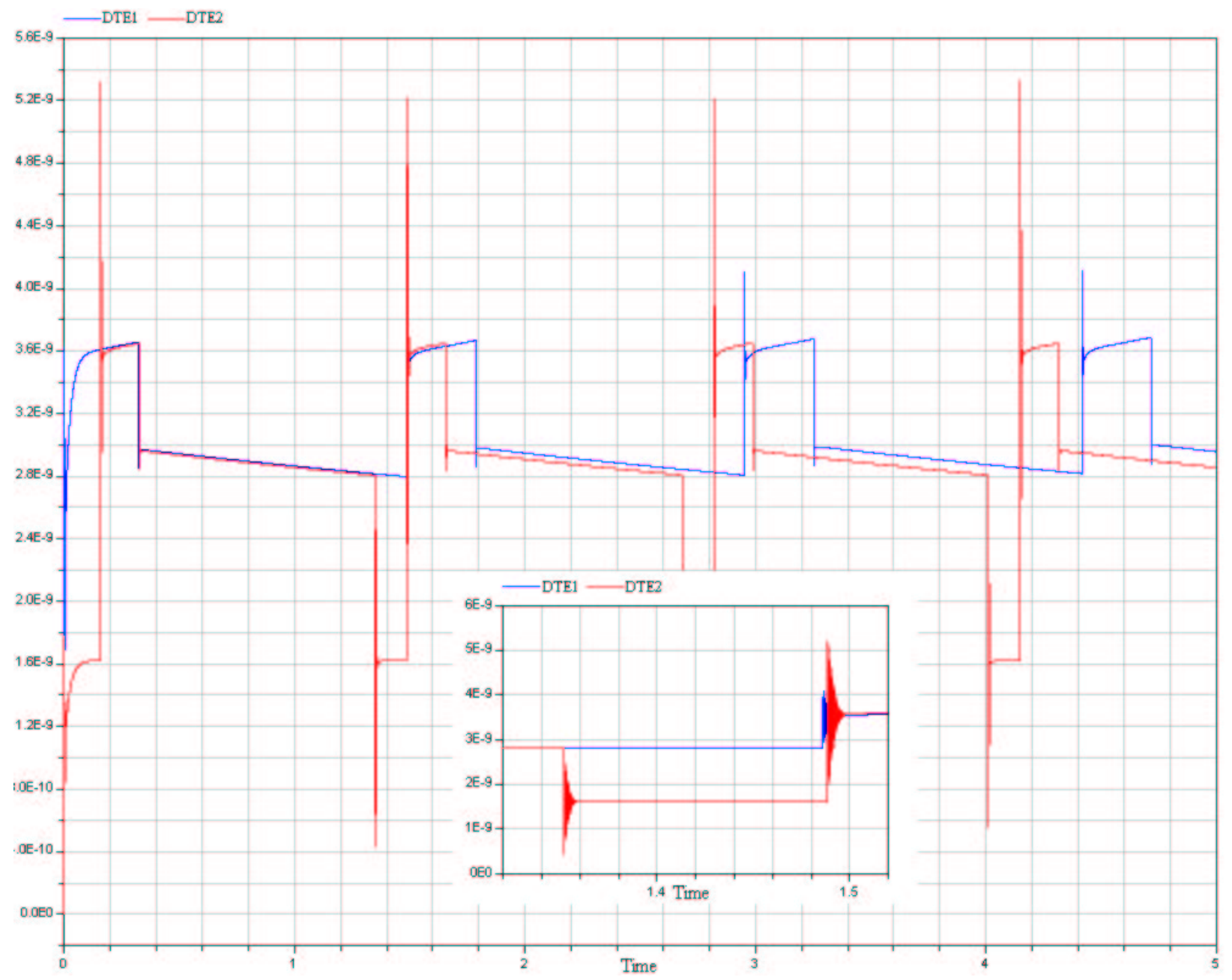

Figure 10: Dynamical transmission error

is implemented using the technique of event processing.

Namely, in usual mode if $\varphi_{\alpha}(t) \in\left[\varphi_{\alpha \min }, \varphi_{\alpha \max }\right]$ then we assume that the value $\varphi_{\alpha}(t)$ satisfies the differential equation

$$
\frac{d \varphi_{\alpha}}{d t}=\frac{d \psi_{\alpha}}{d t} \quad(\alpha=A, B)
$$

where the derivative is supposed to be expressed depending on state variables of the problem. When one of the events: $\varphi_{\alpha}(t)<\varphi_{\alpha \min }$ or $\varphi_{\alpha}(t)>\varphi_{\alpha \max }$ occurs then the initial condition of Cauchy problem is corrected immediately in the corresponding differential equation of the system (4) at the instant $t=t_{*}$ of the event according to the formula

$$
\varphi_{\alpha}\left(t_{*}\right)=\left\{\begin{array}{lll}
\varphi_{\alpha \max } & \text { for } \quad \varphi_{\alpha}\left(t_{*}-\right)=\varphi_{\alpha \min } \quad \text { and } \\
& & \dot{\varphi}_{\alpha}\left(t_{*}-\right)<0, \\
\varphi_{\alpha \min } & \text { for } \quad \varphi_{\alpha}\left(t_{*}-\right)=\varphi_{\alpha \max } \text { and } \\
& & \dot{\varphi}_{\alpha}\left(t_{*}-\right)>0 .
\end{array}\right.
$$

The technique of event processing outlined above provides us with the correct model for simulating physical switching for teeth at contact for the gearbox model simulation.

\section{Numerical experiments}

To verify an improved model of the gearbox numerical experiments were performed similar to those of the work [3]. Graphs for the dynamical transmission error (DTE) and value of the normal elastic force at contact were under verification. For DTE the current model clarifies the time dependence tracking as it has been done in $[9,10]$ instants for increasing/decreasing of the contact multiplicity. One can observe splashes of the value under observation, DTE here, as it was observed also in $[9,10]$ at these moments, see graph of DTE in Figure 10.

The DTE graph for the previous model from [3] is represented here by the blue curve (variable DTE1): the mesh multiplicity is equal to one, and then the teeth contacts overlapping is absent. The red curve (variable 


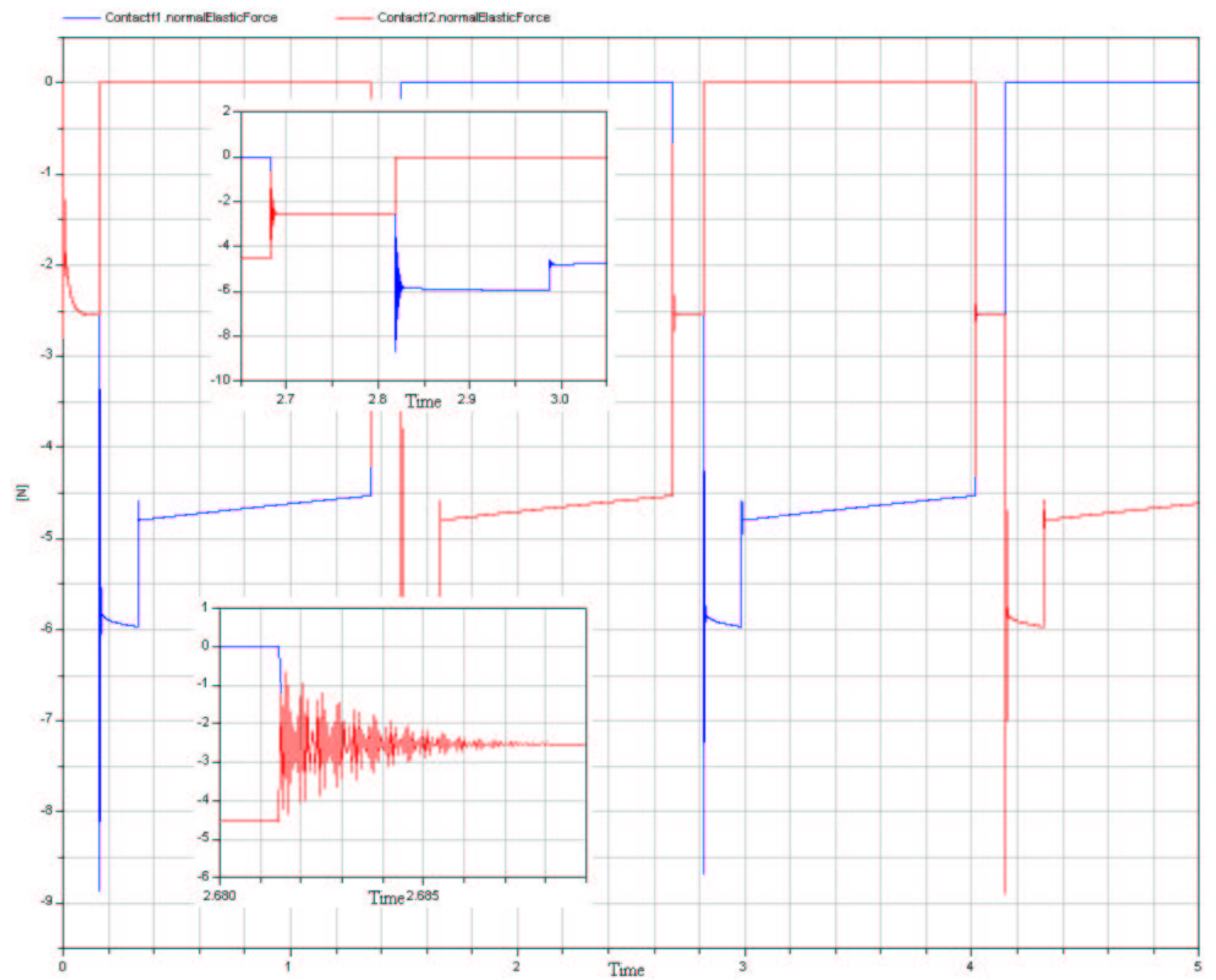

Figure 11: Normal forces at contacts

DTE2) corresponds to the case of the mesh multiplicity being equal to two. In this case there are time intervals for two simultaneously existing contacts, see subplot zoomed in in Figure 10. Interval of two contacts begins with the left splash. Then DTE instantly decreases because total contact stiffness increases with two contacts. Right splashes correspond to the instant of the old contact vanishing. Then only new contact remains. Anyway, in the case under description there exists an overlapping in time for contacts. And yet another observation: as one can also see from the graph an effect of overlapping causes a systematic shift of the mesh cycle. Indeed, total duration of each individual contact mesh cycle remains the same as it was in case of unit multiplicity. At the same time the periodicity in meshing for the case of two contacts becomes shorter by the duration of ovelapping interval.

When exploring a behavior of the normal elastic force we can observe yet another interesting phenomenon. Usually following an engineering tradition one applies the so-called restricted formulation of dynamical problem with multiple contacts: for computing the normal elastic forces at each contact one simply divides the total elastic force by the number of contacts being currently in action, see for instance $[9,10]$. In our current approach, on the contrary, we compute normal elastic force at each contact individually from dynamics and with the use of the Johnson contact model. So one may say that we have implemented soto-speak unrestricted problem for teeth contact of the spur involute gear. The normal contact force behavior along the mesh cycle is shown in Figure 11.

In the Figure 11 one exhibits the time dependence for elastic forces being generated in two different contact objects of the model. One assumes in the case under analysis that the constant accelerating torque acts upon the pinion $A$, while the gear $B$ is under the torque of viscous resistance which is proportional to the value of the wheel angular velocity. The graph presents us yet another interesting, though quite nat- 
ural, observation: an engineering approach which has been described above is indirectly verified by the exact dynamical model with an unrestricted contact model - values of normal elastic forces for contacts concurrently existing turned out to be almost identical. This observation takes place at least for the case of large contact stiffness corresponding to the steel our gearwheels are supposed to be made of.

\section{Conclusions}

Comparing results obtained in [3] with results of current work we can highlight the following properties:

1. The model is capable of simulating both the forward stroke and reversal of the gearbox taking into account a possibility of backlash between teeth.

2. The model is capable of simulating the involute mesh with multiple contacts.

3. The most effective, for the case of involute as tooth profile, contact tracking algorithm is implemented in the model. All this is due to differential or algebraic equations were excluded from the model, and only direct computations were in use.

4. To ensure an accuracy of the model of contact the most suitable implementation turned out to be an array of contact objects. Coordination of their behavior is provided by proper selection of initial conditions for the object variables.

\section{Acknowledgements}

The paper was prepared with partial support of Russian Foundation for Basic Research, projects: 11-0100354-a, 12-01-00536-a, 12-08-00637-a.

\section{References}

[1] Ziegler, P., Eberhard, P. Simulation of Geartrains with an Elastic Model. In: Proceedings of Multibody Dynamics 2011. An ECCOMAS Thematic Conference, Université catholique de Louvain, Brussels, Belgium, July 4-7, 2011, 11 pp. ISBN 978-2-8052-0116-5.

[2] Pelchen, C., Schweiger, C., Otter, M. Modeling and Simulating the Efficiency of Gearboxes and of Planetary Gearboxes. In: Proceedings of 2nd International Modelica Conference, Deutsches Zentrum für Luft- und Raumfahrt e. V. (DLR), Oberpfaffenhofen, Germany, March 1819, 2002, pp. 257-266.

[3] Kosenko, I., Gusev I. Implementation of the spur involute gear model on Modelica. In: Proceedings of the 8th International Modelica Conference, Auditorium Centre of the Technische Universitat Dresden, Germany, March 20-22, 2011, pp. 315328.

[4] Johnson, K. L.: Contact Mechanics. Cambridge: Cambridge University Press, 2001.

[5] Kosenko, I. I., Loginova, M. S., Obraztsov, Ya. P., Stavrovskaya, M. S. Multibody Systems Dynamics: Modelica Implementation and Bond Graph Representation. In: Proceedings of the 5th International Modelica Conference, arsenal research, Vienna, Austria, September 4-5, 2006, pp. 213223.

[6] Kosenko, I. I. Physically Oriented Approach to Construct Multibody System Dynamics Models Using Modelica Language. In: Proceedings of Multibody 2007, Multibody Dynamics 2007. An ECCOMAS Thematic Conference, Politecnico di Milano, Milano, Italy, June 25-28, 2007.

[7] Fritzson, P. Principles of Object-Oriented Modeling and Simulation with Modelica 2.1. Piscataway, NJ: IEEE Press, 2004.

[8] Kosenko, I., Aleksandrov, E., Implementation of the Contensou-Erismann Model of Friction in Frame of the Hertz Contact Problem on Modelica. In: Proceedings of the 7th International Modelica Conference, Como, Italy, 20-22 September 2009. Francesco Casella, editor. Linköping University Electronic Press, 2009. ISBN 978-91-7393-5135. Linköping Electronic Conference Proceedings, ISSN:1650-3740. DOI: 10.3384/ecp0943, pp. 288-298.

[9] Vaishya, M., Singh, R. Sliding Friction-induced Non-Linearity and Parametric Effects in Gear Dynamics. Journal of Sound and Vibration, Vol. 248, pp. 671-694, 2001.

[10] Vaishya, M., Singh R. Strategies for modeling friction in gear dynamics. Journal of Mechanical Design, Vol. 125, pp. 383-393, 2003. 
